УДК $537.212+537.612$

\title{
Limit Halbach Figure
}

Mikhail A. Nechaev*

Siberian State Technological University

Mira, 82, Krasnoyarsk, 660041

Russia

Received 10.05.2016, received in revised form 01.11.2016, accepted 01.02.2017

The shape of a figure of a system that is built on the principle of Halbach and that has a maximum field at own center is obtained.

Keywords: Halbach cylinder, Halbach sphere, Halbach spheroid, limit Halbach figure.

DOI: 10.17516/1997-1397-2017-10-3-314-319.

\section{Introduction}

Systems of permanent magnets that are uniformly magnetized, and the directions of their magnetization are bound by a certain law, could have interesting magnetic field properties. The emergence of permanent magnets with a giant magnetic anisotropy and with a large coercive force contributed to the practical study of such systems. Such magnets have been created on the basis of rare earth metals in 1960s. Systems that have a nearly uniform field in a certain area of a space, and a field strength is much greater than the saturation induction of a material of magnets, are built of such magnets. These systems include both a so-called Halbach cylinder and Halbach sphere. A magnetical Halbach cylinder was suggested by K. Halbach in the work [1]. In later by analogy with a Halbach cylinder, a magnetical sphere [2] has been constructed by other scientists. This sphere also received the name of Halbach. Of course, similar field properties may be found in systems of uniformly electrically polarized bodies if there are suitable materials. In the present paper, we investigate the question of the most advantageous shape of a figure of a system built on the principle of Halbach.

\section{Halbach figures}

\subsection{Cylinder}

The segments of an electrical Halbach cylinder are the uniformly polarized infinitely long isosceles trapezoidal bodies that are symmetric to each other relatively to the cylinder axis. The working area of a Halbach cylinder is a small neighborhood of the cylinder axis in a cavity that is formed by segments. The angle between the segment polarization and the polar axis of the polar coordinate system the origin of which is located on the cylinder axis and the plane of which is perpendicular to the cylinder axis is given by

$$
\gamma(\varphi)=\gamma_{0}+2 \varphi
$$

*mhnech@yandex.ru

(C) Siberian Federal University. All rights reserved 
where $\varphi$ is a polar angle of the mid of the segmet base. If this equality is satisfied, then the maximum field at the cylinder axis is obtained. In Fig. 1, the arrows indicate the directions of a polarization at $\gamma_{0}=-\pi / 2$. This equality is also valid for a magnetical Halbach cylinder. Then, instead of a polarization, segments would have a magnetization.

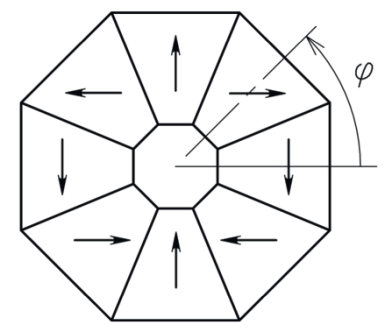

Fig. 1. Cross-section of a Halbach cylinder

The maximum field at the axis of an electrical Halbach cylinder is given by

$$
E_{\max }=4 \pi k P \ln \frac{r_{2}}{r_{1}}
$$

where $k=1 /\left(4 \pi \varepsilon_{0}\right), P$ is the polarization modulus of segments, $r_{1}$ and $r_{2}$ are the inner and outer radii of a Halbach cylinder respectively. The maximum field at the axis of a magnetical Halbach cylinder is given by

$$
B_{\max }=4 \pi k^{\prime} M \ln \frac{r_{2}}{r_{1}},
$$

where $k^{\prime}=\mu_{0} /(4 \pi), M$ is the magnetization modulus of segments.

\subsection{Sphere}

The segments of a magnetical Halbach sphere are the uniformly magnetized truncated right pyramids. The outer surface of a Halbach sphere cover by lower bases of pyramids and is described by a sphere that has the center where the apexes of pyramids are located. The working area of a Halbach sphere is a small neighborhood of the sphere center in a cavity that is formed by segments. In the paper [2] by analogy with a Halbach cylinder, it was assumed that the maximum field at the center of a magnetical Halbach sphere is obtained if the angle between the segment magnetization and the polar axis of the spherical coordinate system of a Halbach sphere is

$$
\gamma(\vartheta)=2 \vartheta
$$

where $\vartheta$ is a zenithal angle of the center of the segment base. This field is codirected to the polar axis. In reality, the dependence $\gamma(\vartheta)$ is another [3].

For a limit Halbach sphere, where a number of segments is infinitely large, we may assume that each segment has the shape of a truncated right circular cone. The field of the uniformly polarized truncated right circular cone at its apex at the polarization $P_{\|}$that is parallel to the cone axis is given by

$$
E_{\|}=2 \pi k P_{\|} \sin ^{2} \alpha \cos \alpha \ln \frac{h_{2}}{h_{1}}
$$


where $\alpha$ is the half-aperture of the cone, $h_{1}$ and $h_{2}$ are the distances between the apex and the cone bases, and $h_{1}<h_{2}$. The field at the apex at the polarization $P_{\perp}$ that is perpendicular to the cone axis is given by

$$
E_{\perp}=-\pi k P_{\perp} \sin ^{2} \alpha \cos \alpha \ln \frac{h_{2}}{h_{1}} .
$$

The solid angle at the apex of the segment-element cone of a limit Halbach sphere is equal to

$$
\sin \vartheta d \vartheta d \varphi=\pi \alpha^{2}
$$

where $\varphi$ is an azimuthal angle of the segment base. Fig. 2 shows such a segment of a Halbach spheroid. Using the equalities (2)-(4), we obtain that the field at the center of an electrical limit Halbach sphere is

$$
E=4 \pi k P \int_{0}^{\pi / 2}\{2 \cos (\gamma-\vartheta) \cos \vartheta+\sin (\gamma-\vartheta) \sin \vartheta\} \sin (\vartheta) d \vartheta \ln \frac{r_{2}}{r_{1}},
$$

where $r_{1}$ and $r_{2}$ are the inner and outer radii of a Halbach sphere respectively. It follows from here that the maximum field at the center of a Halbach sphere is obtained if

$$
\gamma(\vartheta)=\vartheta+\arctan \frac{\tan \vartheta}{2}+\pi \theta\left(\vartheta-\frac{\pi}{2}\right) .
$$

If the equality (1) is used, then we obtain that the field at the center of an electrical limit Halbach sphere is

$$
E=\frac{4}{3} 4 \pi k P \ln \frac{r_{2}}{r_{1}} .
$$

Therefore then the field at the center of a magnetical limit Halbach sphere is

$$
B=\frac{4}{3} 4 \pi k^{\prime} M \ln \frac{r_{2}}{r_{1}} .
$$

And if the equality (6) is used, then we obtain that the maximum field at the center of an electrical Halbach sphere is given by

$$
E_{\max }=\left(1+\frac{\sqrt{3}}{6} \operatorname{artanh} \frac{\sqrt{3}}{2}\right) 4 \pi k P \ln \frac{r_{2}}{r_{1}} \approx 1.38 \cdot 4 \pi k P \ln \frac{r_{2}}{r_{1}} .
$$

Therefore the maximum field at the center of a magnetical Halbach sphere is given by

$$
B_{\max }=\left(1+\frac{\sqrt{3}}{6} \operatorname{artanh} \frac{\sqrt{3}}{2}\right) 4 \pi k^{\prime} M \ln \frac{r_{2}}{r_{1}} \approx 1.38 \cdot 4 \pi k^{\prime} M \ln \frac{r_{2}}{r_{1}} .
$$

\subsection{Spheroid}

Let us obtain a Halbach spheroid by a linear expansion of a Halbach sphere along its polar axis. Let a number of segments of a Halbach sphere tends to infinity, and the dependence (6) is performed for both of these figures. Then the maximum fields at the centers of these figures that made of a same material are equal to each other. Now let a cavity of a Halbach spheroid has the spherical shape and the center of this cavity is located at the center of a Halbach spheroid too (Fig. 2). Then by the formula (5), the maximum field at the center of an electrical Halbach spheroid is

$$
E_{\max }=4 \pi k P \int_{0}^{\pi / 2}\{2 \cos (\gamma(\vartheta)-\vartheta) \cos \vartheta+\sin (\gamma(\vartheta)-\vartheta) \sin \vartheta\} \ln \frac{r(\vartheta)}{r_{1}} \sin (\vartheta) d \vartheta
$$


where $r_{1}$ is the radius of the cavity of a Halbach spheroid, $r(\vartheta)$ is the distance between the center of a Halbach spheroid and the element of its outer surface. The maximum field $B_{\max }$ at the center of a magnetical Halbach spheroid may be expressed analogously.

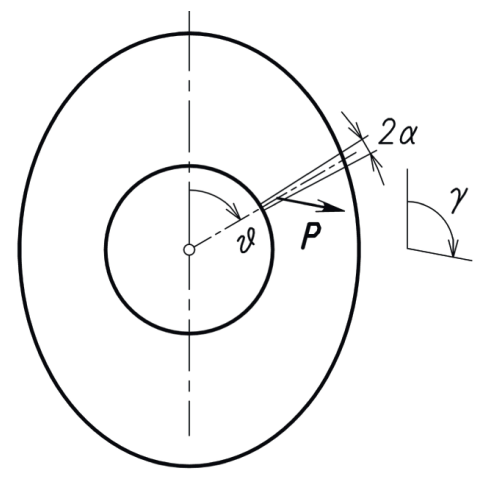

Fig. 2. To constructing of a Halbach spheroid. Here $\alpha$ is the half-aperture of the segment cone

The maximum field at the center of a Halbach spheroid with a spherical cavity can be both more and less than the maximum field at the center of a Halbach sphere that made of a same material and that has a same ratio of volumes of a material and of a cavity as has this Halbach spheroid depending on its a spheroid prolateness. The biggest field is obtained where the prolateness factor equal to about 1.265223. For example, if the ratio of a volume of a material to a volume of a spherical cavity is equal to 10, then the maximum field at the center of a Halbach spheroid that has this prolateness factor is approximately $1 \%$ more than the maximum field at the center of a Halbach sphere.

\subsection{Limit figure}

The problem of the most advantageous shape of a figure of a system that is built on the principle of Halbach is solved analogously to the problem of the shape of a gravitating body that has a maximum field at one point and that has an uniform mass density [4]. Let us use the expression for a field of a dipole that is located on some surface provided that the dependence (6) is performed. The component of a dipole field along the axis of symmetry of this surface at its center should not depend on a point at which this dipole is located. As a result, we obtain that the outer surface of a limit Halbach figure is described by the equation

$$
r^{3} / b^{3}=3 \cos \vartheta[\sin \{\gamma(\vartheta)\} \sin \vartheta+\cos \{\gamma(\vartheta)\} \cos \vartheta]-\cos \{\gamma(\vartheta)\},
$$

where $b$ is the semiminor axis of this surface, $r$ and $\vartheta$ are spherical coordinates of a point on this surface relatively to the center of a limit Halbach figure. And it is not required to know the cavity figure to derive this equation. Therefore the shape of a cavity may be set arbitrarily. Let a cavity has the spherical shape and the cavity center coincides with the figure center. Such a figure is shown in Fig. 3.

Tab. 1 presents the ratios of the maximum field at the center of a Halbach spheroid with a spherical cavity and the maximum field at the center of a limit Halbach figure with a spherical cavity to the maximum field at the center of a Halbach sphere $k_{1}$ and $k_{2}$ respectively that were calculated with the use of the formula (7) at the different ratios $V / V_{c}$, where $V$ and $V_{c}$ are volumes of a material and of a cavity respectively of each of these figures. 


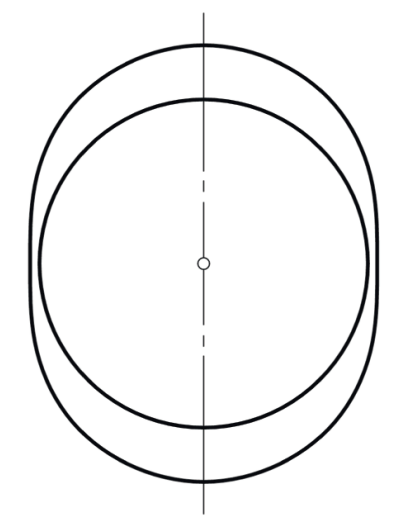

Fig. 3. Axial section of a limit Halbach figure with the concentric spherical cavity at $V / V_{c}=0.5$

Table 1. Ratios of the maximum field at the center of a Halbach spheroid and the maximum field at the center of a limit Halbach figure to the maximum field at the center of a Halbach sphere

\begin{tabular}{|c|c|c|}
\hline$V / V_{c}$ & $k_{1}$ & $k_{2}$ \\
\hline 20 & 1.00763 & 1.00806 \\
10 & 1.00968 & 1.01024 \\
4 & 1.01443 & 1.01525 \\
2 & 1.02113 & 1.02234 \\
1 & 1.03349 & 1.03542 \\
0.5 & 1.05726 & 1.06054 \\
0.3 & 1.08849 & - \\
\hline
\end{tabular}

It should be noted that the values $k_{1}$ and $k_{2}$ differ very small. Therefore, the shape of a spheroid also may be used to construct a most advantageous Halbach figure in practice. And a number of segments can not be very great in practice. Then values $k_{1}$ and $k_{2}$ are slightly smaller. In addition, obtained values $k_{1}$ and $k_{2}$ are close to unity at large values $V / V_{c}$. Therefore, in this case, the spherical shape can be used justly in practice.

\section{Conclusion}

Thus, the shape of a figure of a system that is built on the principle of Halbach and that has a maximum field at own center has been obtained. It is shown that the shape of outer surface of this figure does not depend on a figure of its cavity.

The author thanks Prof. Yu. V.Zakharov for discussions and some of the comments, and Prof. I. V. Yaminsky for the clarification of some details.

\section{References}

[1] K.Halbach, Design of permanent multipole magnets with oriented rare earth cobalt materials, 
Nucl. Instr. Methods, 169(1980), 1-10.

[2] H.A.Leupold, E.Potenziani II, Augmentation of field uniformly and strength in spherical and cylindrical magnetic field sources, J. Appl. Phys., 70(1991), no. 10, part II, 6621-6623.

[3] M.A.Nechaev, On calculation of field of system with uniform polarizations, Izvestiya vysshih uchebnyh zavedenij. Fizika, 51(2008), no. 9/2, 65-69 (in Russian).

[4] E.N.Yunosov, I.V.Yaminsky, Kvant, (1987), no. 10, 31 (in Russian).

\section{Предельная фигура Хальбаха}

Михаил А. Нечаев

Сибирский государственный технологический университет

Мира, 82, Красноярск, 660049

Россия

Получена форма фигуры системы, которая построена по принципу Хальбаха и обладает максимальным полем в своем центре.

Ключевые слова: цилиндр Халъбаха, сфера Халъбаха, сфероид Хальбаха, пределъная фигура Хальбаxa. 